\title{
EFECTOS DEL MARCO REGULATORIO Y LAS DINÁMICAS COMPETITIVAS DE LAS EMPRESAS DE TELEFONÍA MÓVIL EN EL PERU (1993-2018)
}

\author{
Effects of the Regulatory Framework and the Competitive Dynamics of Mobile Telephone
} Companies in Peru (1993-2018)

\begin{abstract}
RESUMEN
La evolución de la telefonía móvil en el Perú desde 1993 hasta nuestros días, a diferencia de otros países fue muy particular, pues su proceso de privatización atravesó un periodo de concurrencia, etapa en la que se restringía la competencia a nuevos operadores, situación que permitió a Telefónica del Perú SA obtener ventajas que fueron sostenidas en el tiempo; sin embargo superada la etapa de concurrencia y sus efectos, el ingreso de nuevas operadoras entre operadoras móviles virtuales y las que cuentan con espectro, así como el nuevo marco regulatorio han posibilitado un escenario de competencia que beneficia a los consumidores.
\end{abstract}

PALABRAS ClAVES: Telefonía móvil marco regulatorio - dinámicas competitivas
Marilia S. Peceros Valencia ${ }^{1}$

\section{ABSTRACT}

The evolution of mobile telephone services in Peru since 1993, unlike other countries, was unusual because its privatization process went through a period in which competition was restricted to new operators, a situation that allowed Telefonica del Peru SA to obtain advantages that were sustained over time; however, after the initial privatization, the entry of new operators, among them virtual mobile operators and those without spectrum, as well as the new Regulatory Law, had made a competitive scenario that benefits customers.

KEY WORDS: Mobile Telephony - Regulatory Law - Competitive dynamics

\footnotetext{
1 Abogada por la Universidad San Antonio Abad de Cusco, egresada de la Maestría en Derecho y Economía de la Universidad de Buenos Aires - Argentina. Trabajó en el Área de Planificación de la empresa VIETTEL PERÚ S.A.C., cuarta operadora de Telecomunicaciones en Perú. Actualmente labora en la Unidad de Planeamiento y Desarrollo del Poder Judicial. Correo electrónico: marilinamx@gmail.com
} 


\section{EVOLUCIÓN DE LA TELEFONÍA MÓVIL EN EL PERÚ}

La telefonía móvil en nuestro país atravesó por varias etapas, al decir de Roxana Cáceres (2005) fueron tres: la estatal, el monopolio y la apertura del Mercado.

En la época estatal, hacia el año 1993, el precio para adquirir una línea costaba alrededor de 2,000 USD a 2,500 USD además del plazo para la instalación que podía tomar entre 4 a 5 años. Por aquellos años se instalaron tres antenas en Lima; en el Morro Solar, en el cerro Centinela de la Molina y en el centro de Lima en el último piso del edificio donde funcionaba el gremio de construcción CAPECO en la Vía Expresa (Comercio E. , 2015); antenas que tenían capacidad para atender una demanda de 1000 líneas.

En 1994, se inició la transferencia de acciones de las empresas ENTEL PERÚ y CTP a TELEFÓNICA DEL PERÚ S.A. En el contrato de concesión se estableció un periodo de concurrencia de 5 años, tiempo en el que se restringía la competencia con la finalidad de que Telefónica del Perú pueda cumplir con las metas de infraestructura. Por lo que hacía 1994 teníamos a TELEFÓNICA DEL PERÚ S.A.A. con $2.1 \%$ y TELE2000 con $3.8 \%$ sobre la cantidad de habitantes (es decir menos del 10\% de la población contaba con teléfono celular, una de cada 100 personas) (OSIPTEL, 2017).

El periodo de concurrencia terminó en 1998, periodo en el que ingresa NEXTEL, posteriormente en 1999 ingresan al mercado las empresas TIM y BELLSOUTH y adquieren el 100\% de las acciones de TELE2000 (Comercio, 2015). Sin embargo, luego de la apertura del mercado y el ingreso de dos nuevas operadoras no se experimentó grandes cambios en la distribución del mercado pues TELEFÓNICA DEL PERU S.A.A. mantuvo el porcentaje más alto de suscriptores entre el 60 al $79 \%$, por 10 tanto, mantuvo su posición de dominio en el mercado del Telefonía Móvil.
La estrategia de ser pionera le permitió a TELEFÓNICA DEL PERU S.A.A. generar ventajas competitivas que no pudieron ser erosionadas sino hasta el año 2013 y el periodo en el que se restringió la competencia le permitió desarrollar ventajas competitivas que pudieron ser mantenidas en el tiempo.

Tabla Nro. 1. Evolución de la Telefonía Móvil en el Perú

\section{Abril 1990}

- La Telefonía Móvil Llega a Perú con TELE 2000

- $\quad$ Se crea CTP Y ENTEL PERU Mayo 1994

- La española MOVISTAR inicia sus operaciones de telefonía móvil.

- Diciembre 1998

- NEXTEL PERU comienza sus operaciones y servicios

- La estadounidense BELLSOUTH CORP acuerda comprar una participación de $38 \%$ de Tele 2000

\section{9}

- BELLSOUTH compra el $100 \%$ de las acciones de TELE2000 y TIM PERU ingresa al Mercado

Marzo 2004

- MOVISTAR adquiere el $100 \%$ de las operadoras de telefonía móvil de BELLSOUTH

\section{Agosto 2005}

- La mexicana América Móvil, compra TIM PERU

\section{Agosto 2012}

- BITEL gana la licitación para obtener Telefonía Móvil en el País

\section{Octubre 2014}

- NEXTEL le vende sus Operaciones a ENTEL CHILE y relanza sus operaciones.

\section{Julio 2016}

- VIRGIN MOVIL inicia sus operaciones.

- TUENTI inicia sus operaciones.

- INKACELL compra las operaciones de VIRGIN MOVIL

- CUY MOVIL, FARMAGUSTA anuncian sus ingresos al mercado peruano. 


\section{VENTAJAS Y SOSTENIBILIDAD DE LA EMPRESA TELEFONICA DEL PERU S.A.}

Telefónica del Perú S.A. ingresó primero al mercado, por lo que es considerada la empresa pionera, la teoría nos dice de las empresas pioneras son las que obtienen ventajas competitivas en costes, diferenciación respecto a las seguidoras, ventajas en recursos humanos y fidelización de clientes que les permiten obtener mayores beneficios y cuotas de mercado. (Urban, 1986)

Las ventajas competitivas en relación a los costes es la inversión que realizan las empresas para prestar el servicio de telecomunicaciones consistente en proyectos de infraestructura como son: despliegue de antenas, instalación de electricidad y cables de fibra óptica, postes, etc. La inversión que realizan es una inversión única que posibilitara la prestación del servicio a un número indeterminado de suscriptores, en este tipo de servicio, la adición de una nuevo suscriptor no ameritara una nueva inversión ${ }^{2}$.

Siendo así la empresa pionera, debido a su permanencia en el mercado ha podido expandir su cobertura en muchos lugares del territorio peruano, esto representa una ventaja debido a su cobertura lo que le permitirá contar con más suscriptores. A diferencia de una empresa entrante que contará con menos cobertura.

Ser pionera en el mercado permite obtener una mayor cantidad de producción acumulada, reduciéndose así los costes unitarios del producto y permitiendo a la empresa una posición de liderazgo en costes respecto a los competidores (Lieberman, 1988).

Otro factor determinante para mantener las ventajas de la empresa pionera, es el efecto del entorno sectorial, es decir las dinámicas competitivas de las empresas que prestan el servicio de telefonía móvil, en el caso peruano, durante los años 1994-1998, se restringió la competencia, situación que fortaleció a la empresa entrante.

En esta misma línea, Covin explica, que cuanto mayor dinamismo se observa en el sector, menos posibilidades tienen las empresas pioneras de obtener una ventaja competitiva sostenible en consecuencia, en unas condiciones de mayor estabilidad - menor dinamismo-, la empresa pionera tendrá mayores posibilidades de obtener ventajas competitivas por no verse sustancialmente modificadas las condiciones que existían en el momento de su entrada al mercado $^{3}$

Otro aspecto del entorno que se analiza es el tipo de proceso regulatorio que se da en el país donde pretende entrar la empresa pionera. En este sentido, cuando el desarrollo del sector está afectado por las elecciones discrecionales de los gobiernos de cada país y existe una convergencia de intereses entre la empresa pionera y otros agentes nacionales, se pueden crear convenios exclusivos, cerrando el mercado a potenciales consumidores, lo que le permite al primer entrante conseguir y sostener ventajas estratégicas y económicas (Garcia Villaverde y Ruiz Ortega, 2006).

\section{EL MARCO REGULATORIO ACTUAL DEL SECTOR DE TELEFONIA MOVIL}

\subsection{La Nueva Constitución Política del Perú del año de 1993}

En el capítulo relativo al Régimen Económico, la constitución establece que el Perú posee una economía social de mercado donde la iniciativa privada y las leyes de la oferta y la demanda

\footnotetext{
${ }^{2}$ Es decir, la inversión que realiza una operadora de telecomunicaciones para la prestación del servicio de una línea telefónica es la misma que efectúa para la prestación del servicio de cien líneas, por lo que a mayor número de suscriptores los costos unitarios de producción disminuirán considerablemente.

${ }^{3}$ Por lo que su planteamiento inicial seguirá siendo el más adecuado para las condiciones actuales.
} 
tienen pertenencia, de modo que el Estado interviene para complementar su funcionamiento y ofrecer seguridades (Chirinos Soto, 2005).

\subsection{Texto Único Ordenado de la Ley de Telecomunicaciones-TUO}

El TUO de la Ley de Telecomunicaciones, establece en su artículo $6^{\circ}$, que el Estado peruano fomenta la libre competencia en la prestación de los servicios de telecomunicaciones ${ }^{4}$. Con este artículo el Estado en concordancia con los principios constitucionales, promueve la competencia entre las empresas privadas que prestan el servicio público de telecomunicaciones, e interviene para regular las malas prácticas de las empresas cuando así lo requiriera (Ferres, 2017).

\subsection{Ley de Telecomunicaciones y su reglamento}

La constitución de 1993 contiene dos disposiciones que aluden directamente a la técnica concesional (i) el artículo $66^{5}$ se refiere a los recursos naturales y el artículo $73^{6}$ se refiere a las concesiones sobre bienes de dominio público.

El decreto Supremo N 059-96-PCM, dispuso en su Artículo $2^{7}$ que la promoción de la inversión privada en servicios públicos se realizará a través del otorgamiento de concesiones. Asimismo, el Decreto Supremo N ${ }^{\circ}$ 060-98-PCM, definió a la concesión de servicios públicos como el Acto Administrativo por el cual el Estado otorga a personas jurídicas nacionales o extranjeras la prestación de servicios Públicos por un plazo establecido (Losada, 2004).

Con relación al artículo citado, debemos rescatar los siguientes aspectos relevantes:

i. Para el sector de las telecomunicaciones en el Perú, la concesión constituye un acto jurídico de derecho público, en la medida que los derechos y las obligaciones se plasmaran en un contrato administrativo.

ii. Se otorgará la Concesión Única para la prestación de todos los servicios públicos de telecomunicaciones.

iii. La concesión se perfeccionará mediante la celebración de un contrato, entre la empresa operadora y el Estado peruano, representado en este caso por el MTC.

\subsection{Ley $N^{\circ}$ 30083: Ley que Establece Medidas para Fortalecer la Competencia en el Mercado de los Servicios Públicos Móviles} En primer lugar, en esta Ley se definen a las Operadoras Móviles Virtuales - OMV como actores que han surgido en distintos países para superar la principal barrera a la entrada del mercado móvil, a saber: la escasez de espectro radioeléctrico. Así, los OMV son empresas que operan sin contar con licencia de uso de espectro pero que, a los ojos de los consumidores son equivalentes a una empresa operadora móvil tradicional.

\footnotetext{
${ }^{4}$ Artículo $6^{\circ}$ : "El Estado fomenta la libre competencia en la prestación de los servicios de telecomunicaciones, regula el mercado de forma que se asegure su normal desenvolvimiento, se controle los efectos de situaciones de monopolio, se evite prácticas y acuerdos restrictivos derivados de la posición dominante de una empresa o empresas en el mercado. Igualmente, el Estado fomenta la participación de los usuarios de servidores de telecomunicaciones, en el establecimiento de tarifas y en la prestación y control de estos servicios".

${ }^{5}$ Artículo 66.- Los recursos naturales, renovables y no renovables, son patrimonio de la nación. El estado es soberano en su aprovechamiento. Por su ley orgánica se fijan las condiciones de su utilización y de su otorgamiento a particulares. La concesión otorga a su titular un derecho real, sujeto a dicha norma legal.

${ }^{6}$ Artículo 73.- Los bienes de dominio público son inalienables e imprescriptibles. Los bienes de uso público pueden ser concedidos a particulares conforme a Ley, para su aprovechamiento económico.

${ }^{7}$ Artículo 2 del Texto único Ordenado de las normas con rango de Ley que regulan la entrega en concesión al sector privado de las obras públicas de infraestructura y de servicios públicos, publicado el 27 de diciembre de 1996.
} 


\subsection{Reglamento para la Supervisión de la Cobertura}

\subsubsection{Portabilidad}

Los principales cambios están orientados a simplificar y automatizar los procedimientos y reducir los costos, esto conllevó a reducir los plazos establecidos para el procedimiento, pasando de un plazo de 7 días a un plazo máximo de 24 horas, salvo los casos en que la solicitud de portabilidad fuera presentada el domingo o feriado, o el día previo a estos. Otro de los cambios fue mantener la continuidad del servicio, para lo cual se dispuso que la interrupción del servicio sería por un periodo máximo de 3 horas, y la ventana de cambio sería de lunes a sábado entre las 0:00 y las 6:00 horas, a excepción de feriados. (Gallardo, 2018).

\subsubsection{Modificación al Reglamento General de Tarifas}

Las modificaciones al Reglamento General se detallan a continuación:

-Se establecieron precisiones de las definiciones ${ }^{8}$ de los distintos tipos de tarifas, distinguiendo en específico entre las Tarifas Establecidas y las Promocionales, así como entre las Tarifas Comercializadas y las Vigentes.

- Se añadieron reglas para la aplicación de Tarifas Promocionales: Los plazos de vigencia no pueden exceder los 180 días calendario de forma continua o acumulada a través de renovaciones en un periodo de 12 meses consecutivos.

-La facturación debe realizarse de manera tal que permita la identificación de las tarifas individuales aplicables a cada uno de los servicios contenidos en el paquete y la tarifa individual que se aplique por la contratación de cada uno de los servicios en forma separada.

\subsection{Nuevo Reglamento de Calidad}

Se establecieron nuevos porcentajes de velocidad mínima, así como aspectos relacionados con los indicadores de servicio móvil de voz y servicios de mensajería, que se detallan a continuación.

\subsubsection{Velocidad Mínima}

Las empresas operadoras están obligadas a prestar el servicio acorde con las velocidades contratadas, así se definió que la velocidad mínima correspondería al $40 \%$ de la velocidad máxima contratada de subida y bajada ${ }^{9}$ Este indicador debe ser cumplido por las empresas operadoras en el $90 \%$ de las mediciones ${ }^{10}$.

\subsubsection{Indicadores del Servicio Móvil de Voz}

Se estableció que el nuevo nivel de desagregación para la medición de Tasa de Intentos No Establecidos (TINE) y de Tasa de Llamadas Interrumpidas (TILLI) sería departamental ${ }^{11}$. Además, se definieron los

\footnotetext{
${ }^{8}$ El artículo $3^{\circ}$ de la Reglamento General de Tarifas, establece las siguientes definiciones:

Tarifa Establecida. - Tarifa que, bajo el régimen de tarifas supervisadas, es determinada libremente por cada empresa operadora para ser aplicada de manera regular y por tiempo indefinido en la prestación de un servicio público de telecomunicaciones, cuya vigencia debe mantenerse hasta que la misma empresa operadora decida modificarla de conformidad con las disposiciones del presente reglamento. En el caso que se trate de servicios sujetos al régimen de tarifas reguladas, la Tarifa Establecida será determinada en virtud del esquema regulatorio particular correspondiente. Las Tarifas Establecidas sirven de base para la aplicación de Tarifas Promocionales.

Tarifa Promocional. - Tarifa que se aplica por elección expresa de los abonados y/o usuarios, bajo condiciones económicas más ventajosas a las regularmente aplicadas en las correspondientes Tarifas Establecidas, y que está sujeta a un período temporal de duración, tanto para su comercialización como para su vigencia de aplicación efectiva. Las Tarifas Promocionales deben estar asociadas a las Tarifas Establecidas que les sirven de base.

${ }^{9}$ Es importante precisar que previamente las empresas garantizaban una velocidad mínima correspondiente al $10 \%$ de la velocidad máxima contratada; no obstante, esa cifra no estaba regulada.

${ }^{10}$ Se ha determinado una gradualidad en su cumplimiento, a saber: $70 \%$ en el primer año, $80 \%$ en el segundo y $90 \%$ en tercer año.

${ }^{11}$ Antes de la aprobación del nuevo Reglamento de Calidad, la medición de estos indicadores era a nivel nacional, con lo cual los problemas en una zona podían atenuarse por un buen desempeño del servicio a nivel nacional. Con la desagregación departamental se busca un servicio de mayor calidad en cada zona.
} 
valores objetivos de los indicadores de Calidad de Cobertura de Servicio (CCS $>=95 \%$ ) y Calidad de $\operatorname{Voz}(\mathrm{CV}>=3.00)$. En caso de incumplimiento, la empresa operadora debe remitir un compromiso de mejora, detallando las acciones a realizar para el cumplimiento de los indicadores de calidad.

\subsubsection{Indicadores del Servicio de Mensajes de Texto}

Para el servicio de mensajes de texto se definieron los parámetros de Proporción de Mensajes de Texto Recibidos (PMTR>=95\%) y Tiempo de Entrega de Mensajes de Texto (TEMT $<=20$ seg.).

\subsubsection{Disponibilidad de los Servicios Finales. -} Se implementó un indicador de calidad vinculado a la Disponibilidad del Servicio calculado de forma semestral. Los valores objetivos del indicador se establecieron en función a la población de los departamentos $\mathrm{y}$ al tipo de servicio.

Además, con el fin de desincentivar la ocurrencia de eventos críticos de amplia duración, se definieron tiempos máximos permisibles para interrupciones de gran afectación: el límite de tiempo ponderado es de 90 minutos para Lima y Callao y de 180 minutos para los departamentos restantes.

\subsection{Modificaciones al Texto Único Ordenado \\ de las Condiciones de Uso}

\subsubsection{Sobre el Servicio de Roaming} Internacional
Se estableció la información mínima a ser proporcionada por las empresas operadoras a los usuarios, cuando estos soliciten la activación del servicio y cuando lo utilicen ${ }^{12}$ y se establecieron dos condiciones para la activación del servicio ${ }^{13}$

\subsubsection{Sobre el Desbloqueo de Equipos Terminales Móviles}

La empresa operadora no podrá condicionar la contratación de los servicios móviles a plazos forzosos. De este modo, los contratos de servicios móviles serán siempre a plazo indeterminado (Art. 15º.

\subsubsection{Respecto a los equipos terminales}

La empresa operadora no deberá restringir o degradar el acceso a los servicios que ofrece, a aquellos usuarios que hayan adquirido equipos terminales de un proveedor distinto de la empresa operadora, siempre que dichos equipos se encuentren debidamente homologados y sean compatibles con la red y tecnología implementadas por la empresa operadora. De igual forma, la empresa operadora no podrá comercializar equipos terminales que tengan alguna restricción de acceso a la red de otro operador.

\subsubsection{Contratos adicionales para la provisión del equipo terminal}

El contrato de prestación del servicio es independiente del contrato adicional celebrado para la provisión del equipo terminal salvo que la empresa operadora otorgue al abonado un beneficio económico para la adquisición del equipo terminal, el cual deberá encontrarse vinculado al valor de la renta fija periódica por la

\footnotetext{
${ }^{12}$ Artículo $6^{\circ}$. - Información básica a ser proporcionada por la empresa operadora “... (xii) Las condiciones de prestación del servicio de roaming internacional, las tarifas aplicables para los destinos frecuentes y, de ser el caso, para las zonas de frontera, así, así como el procedimiento para su activación y desactivación; (...)".

${ }^{13}\left(\right.$ Art. $\left.21-\mathrm{A}^{\circ}\right)$. En primer lugar, se determinó que esta solo se haría efectiva en el momento y por el plazo en que el usuario lo solicitara expresamente. De esta manera, se eliminó la posibilidad de una "activación automática" del servicio, la cual ocasionaba quejas de facturación recurrentes. En segundo lugar, se determinó que la activación se haría efectiva en un plazo no mayor a 24 horas, y que sería gratuita.
} 
prestación del servicio y/o al cumplimiento del plazo de permanencia establecido en el contrato adicional.

Para el caso en que el abonado decida resolver el contrato de prestación del servicio y consecuentemente se resuelva el contrato adicional antes del cumplimiento del plazo de permanencia, la empresa operadora no podrá cobrar al abonado un importe superior al resultado del cálculo que sea realizado en base a la siguiente fórmula ${ }^{14}$ : PENALIDAD = $\left(\mathrm{PETPt}^{=0}-\mathrm{PETS}\right) *(\mathrm{MR} / \mathrm{MP})$

\subsection{Sobre Temas de Seguridad, Información y Derechos de los Consumidores}

- Se estableció un sistema de "Alerta" para consumo de Internet en móviles, de esta manera la empresa operadora está obligada a remitir una alerta a sus usuarios postpago y control, cuando estos hayan consumido el $80 \%$ de lo contratado. - Se reguló la conservación del número telefónico en caso de baja del servicio o cambio de número, siendo así se estableció que la empresa operadora está impedida de asignar el número por 90 días calendario.

- Se estableció el bloqueo de equipos terminales reportados como robados o perdidos, de esta manera la empresa operadora está obligada a suspender el servicio y bloquear el equipo terminal de forma simultánea cuando el usuario reporte un robo o pérdida (Art. 126 $6^{\circ}$, otorgándole al usuario el código de bloqueo y el número de serie del equipo terminal a ser bloqueado (Art. 125).

- Se determinó la obligación de Contar con Página Web, así las empresas operadoras que superen los 500,000 abonados, están obligadas a contar con página web (Art. 8- $\mathrm{A}^{\circ}$ ), y en ella implementar un aplicativo en el que sus abonados puedan acceder a información personalizada sobre su contrato, solicitudes, migraciones, servicios adicionales y otras prestaciones (Art. $\left.10-\mathrm{A}^{\circ}\right)$.

\subsection{Aspectos que Afectan Indirectamente el Desempeño del Mercado Móvil}

Con la finalidad de establecer un régimen especial y temporal en todo el territorio nacional para la instalación y expansión de los servicios públicos de telecomunicaciones, se aprobó la Ley $\mathrm{N}^{\circ}$ 29022, Ley para el Fortalecimiento de la Expansión de Infraestructura en Telecomunicaciones, que posteriormente fue modificada por la Ley $\mathrm{N}^{\circ} 30228$, el 12 de julio de 2014. En dicha Ley se declara a los servicios de telecomunicaciones como una necesidad pública, por lo que se dictan medidas que promuevan la inversión privada en infraestructura necesaria para la prestación de esos servicios; y eliminen las barreras que impidan llevarlos a cabo. Así, se establece que el uso de las áreas y bienes de dominio público por parte de los operadores de los servicios públicos de telecomunicaciones para el despliegue, mejoras y/o mantenimiento de la infraestructura instalada o por instalarse, es a título gratuito.

Del mismo modo, se establece que las atribuciones y competencias municipales se deben ejercer garantizándose que ninguna exigencia impida o afecte la calidad en la prestación de los servicios de telecomunicaciones. En ese sentido, el nuevo Reglamento de la Ley, aprobado mediante Decreto Supremo No 003-2015MTC, el 17 de abril de 2015, establece una serie de medidas para la instalación de infraestructura en telecomunicaciones ${ }^{15}$.

\footnotetext{
${ }^{14}$ Dónde: "PETPt=0" "es el precio del equipo terminal ofrecido bajo la modalidad prepago, al momento de la contratación. "PETS" es el monto efectivamente pagado por el abonado por el equipo terminal. y "M" es el número de meses restantes para el cumplimiento del plazo establecido en el contrato adicional. "MP" es el número total de meses pactado en el referido contrato.

${ }^{15}$ La Primera Disposición Complementaria Final de la Ley №30228 dispone que el MTC debía adecuar el Reglamento de la Ley $\mathrm{N}^{\circ} 29022$ a las modificaciones establecidas; no obstante, de la revisión efectuada al Reglamento, aprobado por Decreto Supremo No 039-2007-MTC, se advirtió que resultaba conveniente emitir un nuevo Reglamento que establezca disposiciones acordes con las modificaciones efectuadas en la Ley $\mathrm{N}^{\circ} 30228$.
} 
De esta manera se establecido un procedimiento de obtención de Autorizaciones para la instalación de infraestructura que se sujetan a un procedimiento de aprobación automática. Además de establecer facilidades brindadas por los Concesionarios del Servicio Público de Electricidad referidas al aprovisionamiento de energía eléctrica necesaria para brindar servicios públicos de telecomunicaciones.

\section{EFECTOS DINAMICOS DE LAS COMPETENCIAS ENTRE LAS EMPRESAS PIONERAS Y SEGUIDORAS}

\subsection{Expansión de las Redes Privadas Móviles}

- Telefónica. - Fue la primera empresa en introducir el concepto de las redes privadas en el Perú, al lanzar en el año 2006 su servicio denominado "Red Privada Movistar ${ }^{16 "}$ (RPM), que consistía en otorgar una bolsa de minutos libres para realizar llamadas hacia otras líneas de la RPM marcando numeral y un el código RPM asociado a la línea que se deseara contactar.

A diciembre de 2015, la totalidad de planes postpago comercializados por la empresa (cuyas rentas van desde los S/. $29.9^{17}$ hasta los S/. 200) incluyen el servicio de RPM ilimitado, que otorga minutos ilimitados para llamar a móviles de Movistar (todos los postpago y los prepagos que gocen del servicio) y hasta 300 minutos para llamar a fijos nacionales. Para los clientes prepago está disponible una promoción que permite tener RPM ilimitado por un día, pagando S/. 1.00 .

A noviembre de 2018 todos los planes prepago y postpago incluyen el servicio RPM ilimitado.

- América Móvil. - Como respuesta al servicio de RPM de Telefónica, A-Móvil lanzó en julio de 2006 la "Red Privada Claro Empresas (RPC ${ }^{18}$ ) únicamente para clientes corporativos empresariales. Posteriormente, en noviembre 2010, lanzó su familia de planes RPC para el público postpago en general. Luego, en mayo del 2014, con el lanzamiento del servicio 4G a través de los planes postpago "Conexión" y "Conexión Plus", se incluyó a los teléfonos fijos de Claro como parte de los destinos de la RPC y, además, se pasó a ofrecer minutos ilimitados RPC para un mayor número de planes; de tal forma que, a diciembre de 2015, casi la totalidad de planes comercializados cuentan con minutos ilimitados para el referido servicio. Los móviles prepagos no forman parte de la RPC.

A noviembre de 2018, se extiende este servicio a los planes prepago, postpago y fijos claro.

\footnotetext{
${ }^{16}$ En un inicio, este beneficio era exclusivo para clientes corporativos. Posteriormente, entre los años 2007 y 2008 , Telefónica empezó a lanzar planes que contenían el servicio de RPM para grupos de clientes específicos como miembros de la PNP o de la FAP, trabajadores de Essalud, etc. Finalmente, a fines del año 2009, el referido servicio se expandió hacia el segmento residencial, al incluirlo como parte de los beneficios de los planes postpago asociados a rentas mensuales altas.

Las condiciones del servicio se mantuvieron iguales hasta el año 2013, en el que Telefónica añadió a los teléfonos fijos nacionales como parte de los destinos RPM a los cuales se podía cursar llamadas con cargo a la bolsa de minutos otorgada. Además, se amplió la cantidad de planes postpago que podían acceder a este servicio, expandiendo el beneficio hacia planes con rentas mensuales inferiores.

Adicionalmente, en noviembre de 2014, el servicio de RPM se puso a disposición de los clientes prepago que deseasen acogerse al servicio, sujetos a la condición que recarguen un mínimo de 5 soles en el mes. El servicio de RPM prepago, permite llamar a otros RPM o fijos de Telefónica a una tarifa menor que la tarifa establecida del plan.

${ }^{17}$ El plan de renta mínima que brinda el servicio de RPM ilimitado se denomina "Chip 29.90" (con renta mensual de S/.29.90) - con código SIRT TETM201500428.

${ }^{18} \mathrm{Al}$ igual que la RPM de Telefónica, el RPC de A-Móvil consistía en otorgar una bolsa de minutos libres para realizar llamadas, la diferencia radica en que la RPC permitía realizar llamadas a todos los números postpago de Claro (no solo a los que contrataban un plan RPC) y la marcación era de forma directa.
} 
- Entel. - En octubre de 2014, incluyó, como atributo de algunos de sus planes postpago, el servicio de Red Privada Entel (RPE) que otorgaba minutos ilimitados para realizar llamadas hacia móviles de Entel tanto postpago como prepago ${ }^{19}$ y fijos nacionales. Es decir, la RPE abarca todas las llamadas On-net y todas las llamadas a fijos nacionales, otorgando un beneficio superior al de sus pares. No obstante, mientras que en el caso de Telefónica y A-Móvil este servicio está incluido en la mayoría o la totalidad de planes postpago, en Entel este servicio está disponible únicamente para los planes con rentas mensuales desde $\mathrm{S} / .74^{20}$.

-Bitel. - Desde su ingreso al mercado en julio de 2014, lanzó también planes con el servicio denominado "Red Privada Bitel" (RPB) que consiste en otorgar un bono de minutos libres para llamadas a todos los postpago de Bitel. Es decir, su red privada se asemeja a lo que fueron en sus inicios las redes privadas de A-Móvil y Telefónica. Al respecto, cabe mencionar que la bolsa de minutos otorgada en su mayoría ha sido y continúa siendo ilimitada.

\subsection{Acceso a Redes Sociales Ilimitadas}

i. Telefónica. - El beneficio consistía en otorgar a los clientes de altas nuevas de planes postpago comercializados entre abril y mayo de 2013 , un bono temporal para hacer uso de "Facebook Ilimitado" por un periodo de hasta de 3 meses. Por otro lado, tras el lanzamiento de su marca Tuenti en octubre de 2014, Telefónica amplía este beneficio para los clientes prepago de dicha marca. Así, lanzó "combos" desde S/. 5 que incluían WhatsApp ilimitado como beneficio adicional a su paquete de minutos, megabytes y SMS contratados. Adicionalmente, lanzó el "X- tra" que otorga Facebook + Facebook Messenger ilimitados por 1 día por $\mathrm{S} /$. 1 .

Del mismo modo, en mayo de 2015, Telefónica lanzó su promoción "Supercargas ilimitadas" que permiten acceder a WhatsApp ilimitadamente por un día pagando $\mathrm{S} /$. 1. Este beneficio está dirigido a clientes prepago de Movistar. Posteriormente, lanzó su promoción de Facebook Ilimitado" que permite acceder a esta red social de forma ilimitada durante un día por el pago de S/. 1, y su promoción "Facebook + WhatsApp + Twitter Ilimitado" que permite acceder a WhatsApp, Facebook y Twitter ilimitadamente por una semana pagando $\mathrm{S} / .6 .90$ o por 15 días pagando S/. 14.90. Posteriormente, en octubre de 2015 lanzó su promoción "WhatsApp Ilimitado x 4 meses" que permite a los clientes del plan "Superchip ${ }^{21}$ " obtener el beneficio de acceso ilimitado al WhatsApp por los 4 primeros meses.

En setiembre de 2018, lanzó su promoción SMS ilimitado, WhatsApp ilimitado, Facebook ilimitado, por S/. 1. Sol, y con vigencia de un día o S/. 6.90 por 07 días.

- América Móvil. - A-Móvil comenzó a ofrecer el servicio de WhatsApp gratis hacia fines de 2014 como beneficio adicional para altas nuevas de los planes "RPC Ilimitado". Posteriormente, en febrero de 2015, extendió este beneficio para clientes de la competencia que se portaran hacia los planes "Conexión Plus". Finalmente, a fines de febrero, extendió este beneficio para todos sus clientes independientemente de la modalidad contractual y de su condición (clientes portados, altas nuevas, o clientes actuales) y con la única condición para sus clientes prepago de mantener un saldo mínimo de S/. 1 en la cuenta principal.

\footnotetext{
${ }^{19}$ Para que el tráfico cursado desde una línea postpago o control hacia un prepago sea deducido de la bolsa RPE, esta última deberá haber realizado una recarga mínima de S/.20 dentro del mes

${ }^{20}$ En julio de 2015, Entel lanzó el plan “Entel Chip RPE 74” que es el de menor renta mensual que incluye el servicio RPE como atributo.

${ }^{21}$ El plan "SuperChip" es comercializado solo por algunos canales de venta, y está disponible únicamente para clientes prepago que hayan adquirido en las últimas 24 horas un Chip Movistar y hayan efectuado una recarga mínima de 8 soles.
} 
Por otro lado, A-Móvil lanzó los planes postpago "Conexión" (en junio de 2015) y "Conexión Chip" (en julio de 2015), los cuales, a partir de rentas mensuales de S/. 69 y S/. 59, respectivamente, ofrecen acceso ilimitado para redes sociales (WhatsApp, Facebook y Twitter) como beneficio extra a los servicios incluidos en cada plan.

Asimismo, para aquellos clientes postpago que no cuenten con el servicio de redes sociales dentro de su plan, ofrece la posibilidad de adquirir los paquetes mensuales "WhatsApp Ilimitado" (por S/. 15) o "Redes Sociales (Facebook y Twitter)" (por S/. 20); y, para los clientes prepago ofrece el paquete diario de "Facebook + Twitter" desde S/. 1.

Cabe precisar que el servicio de WhatsApp ilimitado brindado por A-Móvil, inicialmente estaba restringido al envío de mensajes de texto. Sin embargo, desde el 28 de febrero de 2015, el servicio incluye además el envío y descarga de contenidos como videos, fotos, notas de voz y compartición de contactos de la agenda, además de la recepción de notificaciones ${ }^{22}$.

En agosto de 2018, A-móviles lanzo la promoción de acceso a Instagram, WhatsApp y Facebook ilimitado por S/. 1 sol con una vigencia de un día.

- Entel. - Por su parte, desde agosto de 2015, Entel ofrece por S/. 1 el servicio de WhatsApp ilimitado por un día para determinados clientes control (actuales y altas nuevas) y prepago; y la promoción "Superyapa Potenciada Prepago" que otorga 30 minutos todo destino y WhatsApp ilimitado para aquellos clientes prepago que realicen una recarga mínima de $\mathrm{S} / .5$, con una vigencia de 3 días.

Por otro lado, a partir de setiembre de 2015, la empresa lanzó una promoción, para un total de 100 planes ("Entel Libre, Control y Chip" entre otros), que permite acceder de forma gratuita e ilimitada a las aplicaciones Facebook y Facebook Messenger a través de la aplicación o página web Free Basics.

Desde setiembre de 2018, Entel lanzo, el acceso ilimitado a Facebook, Messenger Facebook, Instagram, WhatsApp, iMusic, Wase, Spotify para sus planes postpago de S/. 109 soles y S/. 129 soles.

- Bitel. - Ofrece el servicio de WhatsApp ilimitado para los clientes de sus planes prepago "Bifri" (desde enero de 2015), "Ahorra", "Ganga" y "Nuevo UNI2" (desde marzo de 2015), siempre que realicen como mínimo una recarga mensual de $\mathrm{S} / .3 ; \mathrm{y}$, recientemente, a partir de noviembre, se ha incluido el servicio de Facebook ilimitado por siete días, para clientes prepago de los referidos planes que realicen una recarga mínima de $\mathrm{S} / .5$.

En noviembre, lanzo la promoción recarga S/. 10 soles y recibe, llamadas ilimitadas hasta con 50 contactos, WhatsApp y Line Ilimitado, YouTube ilimitado de 00:00 horas -05:00 horas.

\subsection{Lanzamiento del servicio de Internet con tecnología 4G}

El 22 de julio de 2013 los postores T-Móviles y Americatel (filial del grupo chileno Entel) se adjudicaron los Bloques $\mathrm{A}$ y $\mathrm{B}$, respectivamente, de la Banda de 1.7/2.1 GHz (Banda AWS), banda especializada para soportar la tecnología 4G LTE.

Tras la adjudicación, la primera empresa en lanzar planes postpago con tecnología 4G LTE (en adelante, planes 4G) fue Telefónica, quien en noviembre de 2013 empezó la pre-venta de sus planes 4G denominados "Vuela", habilitando el servicio de Internet 4G LTE desde el primero de enero de 2014.

A pesar de no haberse adjudicado ningún bloque de la referida subasta, la segunda empresa en brindar el servicio de Internet 4G LTE fue A-

${ }^{22}$ Códigos SIRT TPTM201500146 o TPTM201500162 
Móvil, en mayo de 2014, a través de los planes "Conexión" y “Conexión Plus". Para ello, realizó modificaciones técnicas, con el objetivo de habilitar la red 4G sobre la banda $1900 \mathrm{MHz}$ que posee.

Por último, Entel inició la comercialización de planes 4G en octubre de 2014 (fecha de inicio de operaciones comerciales bajo la marca Entel), a través de los planes "Entel Libre" y "Entel Control".

El lanzamiento de los nuevos planes $4 \mathrm{G}$ no solo significó el incremento de las velocidades para los clientes postpago, sino que también vino acompañado de un incremento de velocidades para los clientes prepago. Además, trajo consigo mayores atributos entre los que destacan el acceso a aplicaciones gratuitas y el incremento de las capacidades de transmisión de los paquetes de datos.

\subsection{Comercialización de Equipos Terminales} Hasta el año 2014, la estrategia comercial adoptada por los operadores móviles era la de comercializar planes postpago con acuerdos de adquisición de equipos móviles. De esa forma, a mayor plazo forzoso y/o renta mensual del plan, se podía obtener un mayor descuento sobre el precio de venta del equipo.

Si bien la referida estrategia fue adoptada también por Bitel y Entel desde el inicio de sus operaciones comerciales, Entel, aprovechando el relanzamiento de la portabilidad numérica móvil, empezó a ofrecer descuentos adicionales en los equipos a clientes de la competencia que decidieran portarse a un plan postpago. En respuesta a ello, los demás operadores redujeron los precios de sus equipos, haciendo que incluso equipos de alta gama tuvieran un precio de $\mathrm{S} / .1$ asociados con determinados planes de altas rentas mensuales.
Por otro lado, Telefónica desarrolló una modalidad especial de canje de equipos terminales, la cual daba a sus clientes (desde finales de 2014) y clientes de la competencia que decidieran portarse a Telefónica (desde marzo de 2015), la posibilidad de canjear algunos modelos de equipos terminales antiguos por equipos terminales nuevos (siempre que estos se encontraran en buenas condiciones).

Adicionalmente, lanzó el "Programa Inteligente" el cual permitía renovar anualmente el equipo terminal por la última versión disponible en el mercado, para aquellos clientes que contrataran el Plan "Vuela Inteligente" cuya renta mensual es S/. 199.9023. Por su parte, en julio de 2015, AMóvil desarrolló un nuevo esquema de adquisición de equipos terminales, el cual permite financiar el importe de los equipos terminales hasta en 24 cuotas mensuales.

Ahora bien, la posibilidad de terminar el contrato con un operador pagando una penalidad, y las mayores facilidades para la portabilidad, han generado que durante el 2015 los operadores móviles hayan lanzado planes que permiten al usuario contratar un plan no asociado con la adquisición de un equipo. En ese sentido, AMóvil lanzó los planes "Conexión Chip", disponibles sin acuerdos de adquisición de equipos, los cuales permiten un ahorro en la renta mensual de hasta $\mathrm{S} / .40^{24}$ en comparación a los planes ligados al subsidio de equipos terminales.

Telefónica, Bitel y Entel también cuentan con planes que no involucran la adquisición de equipos terminales, pero que permiten un ahorro en la renta mensual. Para el caso de Telefónica, estos planes se denominan "Vuela Ahorro", lanzados en mayo de 2015, y permiten un ahorro de hasta S/. 2025. En el caso de Bitel, este operador cuenta con los planes "IChip", lanzados

\footnotetext{
${ }^{23}$ El importe incluye el costo asociado al plan ("Vuela Inteligente" que por una renta de S/. 199.90 otorga RPM ilimitado, minutos todo destino ilimitados, SMS ilimitados y $3072 \mathrm{MB}$ ) y el seguro Movistar.

${ }^{24}$ En particular, el Plan Claro Conexión Chip 99 ofrece las mismas prestaciones que el Plan Claro Conexión S/. 139, estando este último asociado a la adquisición de un equipo.
} 
en junio 2015 y permiten ahorros de hasta $\mathrm{S} /$. $20^{26}$. Finalmente, en el caso de Entel, existen planes denominados "Entel Chip", lanzados en julio 2015, los cuales permiten un ahorro de hasta S/. $90 .{ }^{27}$

\section{CONCLUSIONES}

1. Luego de efectuar el análisis de las normas emitidas a partir del año 2013, en materia de telefonía móvil se puso de manifiesto que el marco regulatorio de telefonía móvil, logró dinamizar el Mercado Móvil, haciendo un fuerte contraste con los resultados de los años previos.

2. Las disposiciones normativas estudiadas fueron: Las Medidas complementarias para la aplicación de la Portabilidad numérica, el Reglamento de Tarifas, el Nuevo Reglamento de Calidad, el Texto Único Ordenado de las Condiciones de Uso, la Ley de Promoción de la Banda Ancha y Construcción de la Red Dorsal Nacional de Fibra óptica, el reglamento de la Ley para el fortalecimiento de la Expansión de Infraestructura en Telecomunicaciones. Posibilitaron desarrollar estrategias comerciales a las empresas entrantes, logrando erosionar las ventajas competitivas de la empresa pionera.

3. Después de muchos años de dominio de los grandes operadores, finalmente el índice de concentración alcanzó su nivel histórico más bajo. Así, al cierre de 2017, los relativamente jóvenes Entel y Bitel se apoderaron del
16,3\% y 13,5\% de participación del mercado, respectivamente, según datos del ente regulador Osiptel. En tanto, Movistar registró una fuerte caída de 6,5 puntos y se quedó con el $38 \%$ de la torta, mientras que Claro obtuvo el $32 \%$. Atrás quedaron los años en que estos dos operadores dominaban el mercado con participaciones que superaban el $40 \%$ o $50 \%$.

4. Así, en el corto plazo, se espera que tanto Entel como Bitel sigan ganando participación, y se espera también el ingreso de los Operadores Móviles Virtuales. Con ello, se presentará mayor competencia y desconcentración en el mercado. Producto de ello, se espera que los operadores lancen más ofertas comerciales que beneficien a los usuarios y dinamicen el mercado, como las que se han venido observando en los últimos meses. Del mismo modo, se espera que los servicios móviles se sigan expandiendo en más distritos del país.

5. El ingreso de nuevas operadoras fomento la competencia entre las mismas, y el ganador neto fue el consumidor. En la actualidad hay registradas 110 líneas por cada 100 habitantes haciendo un contraste con los años 1993, cuando por cada 100 habitantes solo 1 accedía a este servicio.

6. En cuanto a uno de los efectos de las dinámicas competitivas entre la operadora, se tiene que a julio de más de 6 millones de personas migraron de operadoras y la perdieron neta fue movistar que perdió 3203 , 274 líneas.

\footnotetext{
${ }^{25}$ Por ejemplo, el plan Vuela Ahorro con una renta mensual de S/. 90 ofrece $2 \mathrm{~GB}$ de internet, mientras que para alcanzar el mismo nivel de datos en un plan Vuela (asociado a la adquisición de equipo), se requiere contratar un plan con una renta mensual de $\mathrm{S} / .110$.

${ }^{26}$ Por ejemplo, el plan "IChip" asociado a una renta mensual de S/. 69.90 reduce la velocidad tras alcanzar 2.5 GB de descarga, mientras que el plan MegaPlus que ofrece ese nivel de datos antes de reducir la velocidad de descarga es el plan MegaPlus asociado a una renta mensual de S/. 89.90.

${ }^{27}$ El ahorro puede ascender hasta un máximo de S/. 90 si se realiza la comparación entre el plan control "Entel Chip 209" (solo chip) y postpago "Entel Libre 299" (equipo más chip), los cuales presentan los mismos beneficios y cuya única diferencia es que el primer plan no se encuentra asociado a la adquisición de equipos, mientras que el segundo sí. 


\section{REFERENCIAS BIBLIOGRÁFICAS}

-CÁCERES BARRANTES, R. (2005). La Regulación para el desarrollo de las Telecomunicaciones en Perú 1993-2001. JCAS Ocasional Paper $\mathrm{N}^{\circ} 25$.

-GARCIA VILLAVERDE, G y L. RUIZ ORTEGA (2016). Marker Share Reward to pioneering Brands: As Empirical Analysis and Strategic Implications, Management Since.

-Chen, M. (1996). Competitor analysis and interfirm rivalry: Toward a theoretical Integration. Maryland: Academy of Management Journal.

-Comercio, D. e. (06 de 02 de 2015). El comercio.pe. Recuperado el 2018 de 09 de 12, de ElComercio.pe:

https://elcomercio.pe/economia/negocios/histori a-revolucion-telefonia-movil-peru-330906

-Comercio, E. (06 de 02 de 2015). La historia de la revolución de la telefonía móvil en el Perú. El Comercio, pág. 23.

-Garcia Villaverde, P. \&. (2000). La Estrategia de ser Pionera. Mexico: Red de Revistas Cientificas de América Latina y El Caribe, España y Portugal.

-Golder, P. \&. (1993). Pioneer advantage: Marketing logic or marketing legend? Mexico: Journal of Marketing Research.
-Ley 30083-Ley que Establece Medidas para Fortalecer la Competencia en el Mercado Móvil. Obtenido de OSIPTEL: https://www.osiptel.gob.pe/articulo/ley-30083fortalecer-competencia-servicios-moviles

-Ley de Telecomunicaciones y su Reglamento Decreto Supremo No 20-2007-MTC. Obtenido de:

http://transparencia.mtc.gob.pe/idm docs/norma s legales/1 0 2137.pdf

-LIEBERMAN, M., \& MONTGOMERY, B. (1998), First movers advantages. Strategic Management Journal.

-OSIPTEL. Obtenido de Libro Balnco sobre la apertura de las telecomunicaciones en el Perú: www.osiptel.gob.pe

-Resolución de Consejo Directivo Nº 024-2014CD/OSIPTEL. Obtenido de OSIPTEL: https://www.osiptel.gob.pe/Archivos/Resolucio nAltaDireccion/ConsejoDirectivo/Res024-2014$\underline{\text { CD.pdf }}$

- Resolución de Consejo Directivo N 123-2014CD/OSIPTEL. Obtenido de OSIPTEL: https://www.osiptel.gob.pe/Archivos/Resolucio nAltaDireccion/ConsejoDirectivo/Res123-2014CD.pdf 